\title{
Classical and quantum radiation reaction for linear acceleration
}

\author{
Atsushi Higuchi ${ }^{1}$ and Giles D. R. Martin ${ }^{2}$ \\ Department of Mathematics, University of York, \\ Heslington, York YO10 5DD, UK \\ email:11ah28@york.ac.uk, ${ }^{2}$ gdrm100@york.ac.uk
}

(Dated: January 5, 2005)

\begin{abstract}
We investigate the effect of radiation reaction on the motion of a wave packet of a charged scalar particle linearly accelerated in quantum electrodynamics. We give the details of the calculations for the case where the particle is accelerated by a static potential that were outlined in Phys.Rev. D 70 (2004) 081701(R) and present similar results in the case of a time-dependent but space-independent potential. In particular, we calculate the expectation value of the position of the charged particle after the acceleration, to first order in the fine structure constant in the $\hbar \rightarrow 0$ limit, and find that the change in the expectation value of the position (the position shift) due to radiation reaction agrees exactly with the result obtained using the Lorentz-Dirac force in classical electrodynamics for both potentials. We also point out that the one-loop correction to the potential may contribute to the position change in this limit.
\end{abstract}

PACS numbers: 03.65.Sq, 12.20.Ds, 41.60.-m

\section{INTRODUCTION}

A charged particle radiates when it is accelerated. This simple discovery has been the basis of a multitude of technological advances since. Most work, however, has concentrated on the radiation itself. The effect of the radiation, which carries energy and momentum, on the particle itself is very small but still present. The overall effect is a change in the equation of motion for the particle. The nature of this modified equation has been subject to much controversy since the initial work of Abraham and Lorentz. The standard equation for the change in the energy and momentum of the particle in classical electrodynamics is the relativistic generalization by Dirac of the work of Abraham and Lorentz and called the Lorentz-Dirac (or Abraham-Lorentz-Dirac) equation [1, 2, 3]. (See, e.g., Ref. [4] for a modern review.) Thus, if a charge $e$ with mass $m$ is accelerated by an external 4force $F_{\text {ext }}^{\mu}$, then its coordinates $x^{\mu}(\tau)$ at the proper time $\tau$ obey the following equation:

$$
m \frac{d^{2} x^{\mu}}{d \tau^{2}}=F_{\mathrm{ext}}^{\mu}+F_{\mathrm{LD}}^{\mu},
$$

where the Lorentz-Dirac 4-force $F_{\mathrm{LD}}^{\mu}$ is given by

$$
F_{\mathrm{LD}}^{\mu} \equiv \frac{2 \alpha_{c}}{3}\left[\frac{d^{3} x^{\mu}}{d \tau^{3}}+\frac{d x^{\mu}}{d \tau}\left(\frac{d^{2} x^{\nu}}{d \tau^{2}} \frac{d^{2} x_{\nu}}{d \tau^{2}}\right)\right] \text {. }
$$

We have let $c=1$ and defined $\alpha_{c} \equiv e^{2} / 4 \pi$. Our metric is $g_{\mu \nu}=\operatorname{diag}(+1,-1,-1,-1)$. This equation of motion is fundamentally different in form from those which one usually encounters in mechanics as it is third-order. This implies that a third initial condition is needed in addition to the position and velocity. Herein lies the cause of much of the debate as to the physical correctness of this theory. It should be noted that there are many derivations of the Lorentz-Dirac force using a number of different methods besides the original work of Dirac [3] (see, e.g., Ref. [5]).

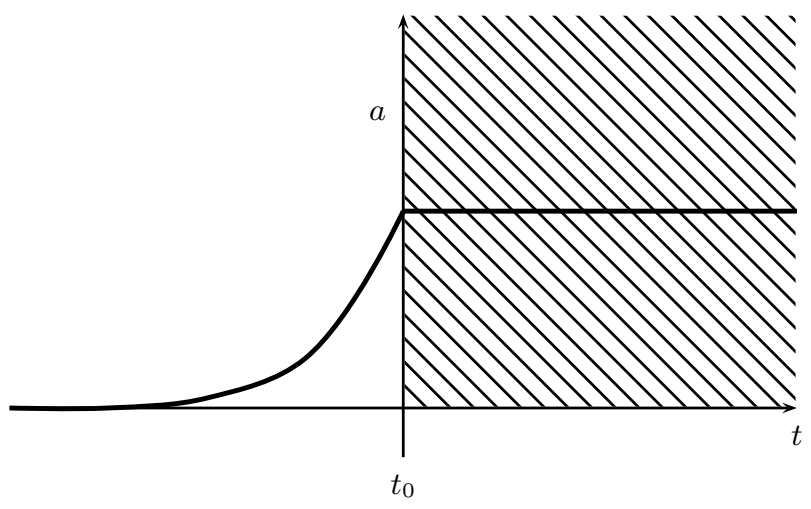

FIG. 1: Preacceleration of a charged particle

However, many unphysical and problematic effects occur in the implementation. One example is the existence of run-away solutions for which a particle will continue to accelerate under its own radiation reaction. This is certainly an unphysical effect and thus a problem for the theory. Alternative forms of implementing the LorentzDirac force, including the integro-differential form, solve the problem of run-aways but create another problem in the form of pre-acceleration. Thus, in this implementation of the Lorentz-Dirac force the particle accelerates before the external force causing the acceleration is applied; this is an acausal effect which should not be present in classical mechanics. This effect is represented in Fig. 11 which shows the acceleration of the particle, $a$, against time, $t$, where a constant force is applied after time $t_{0}$, i.e. in the shaded region.

Additional conditions and procedures are therefore necessary in order to treat the Lorentz-Dirac theory as a a normal causal classical theory, and there are such procedures (see, for example, the review [4] or the discussions in [6] and [7]). One such procedure is the reduction of 
order [8], in which the Lorentz-Dirac force is treated as a perturbation order by order. Despite this, the debate over the controversial aspects of Eq. (1.2) has continued, its impact muted for a couple of reasons. Firstly, the effect due to the third-derivative term, often called the Schott term, is tiny for most physical situations; in fact the time scale for the pre-acceleration is too small for any known classical interactions for it to have a measurable effect. Secondly, the advent of quantum electrodynamics (QED), which is the more fundamental theory of electrodynamics, has rendered the problems concerning the classical Lorentz-Dirac force less urgent. The second point, however, naturally leads to the following question: "If the perturbative treatment of the Lorentz-Dirac force is causal and satisfactory, can it be derived in QED in the $\hbar \rightarrow 0$ limit in perturbation theory?" This question is the main motivation of this paper.

In fact it was found in Refs. 9, 10] (after an initial claim to the contrary) that the change of the position, called the position shift, due to the Lorentz-Dirac force of a charged particle linearly accelerated by a static external potential is reproduced by the $\hbar \rightarrow 0$ limit of one-photon emission process in QED in the non-relativistic approximation. (See, e.g., Refs. [12, 13, 14] for other approaches to arrive at the Lorentz-Dirac theory from QED.) Here we present in detail the generalization of this work to a fully relativistic particle, which was outlined in [11]. At the end of the paper we shall also show similar results with a time-dependent but space-independent potential. The paper is organized as follows. In Secs. III and III we outline the classical and quantum models that are used in the paper. In Sec. IV we calculate expressions for the position expectation value for both the hypothetical non-radiating particle (Subsection IVA) and the radiating particle (Subsection IVB) before identifying the expression for the position shift in terms of the emission amplitude. This amplitude is calculated in Sec. V] The result is used in Sec.VI to show that the energy emitted in the $\hbar \rightarrow 0$ limit in QED is given by the classical Larmor formula and to compute the quantum position shift in Sec. VII The comparison between the classical and quantum position shifts is presented in detail in Sec.VIII and the time-dependent case is presented in Sec. IX] before we conclude the paper in Sec. $\mathrm{X}$

\section{CLASSICAL MODEL}

We start by describing the model to be investigated in this paper. Consider a charged particle with charge $e$ and mass $m$ moving in one space dimension under a potential. Let this motion be in the positive $z$-direction and let the potential be a function of the spatial coordinate $z$, viz $V=V(z)$. We wish to analyze the change in the position of the particle due to radiation reaction. Thus we consider a model in which there has been a period of acceleration, i.e. non-constant potential, at some time in the particle's history. We assume that $V(z)=V_{0}=$ const.

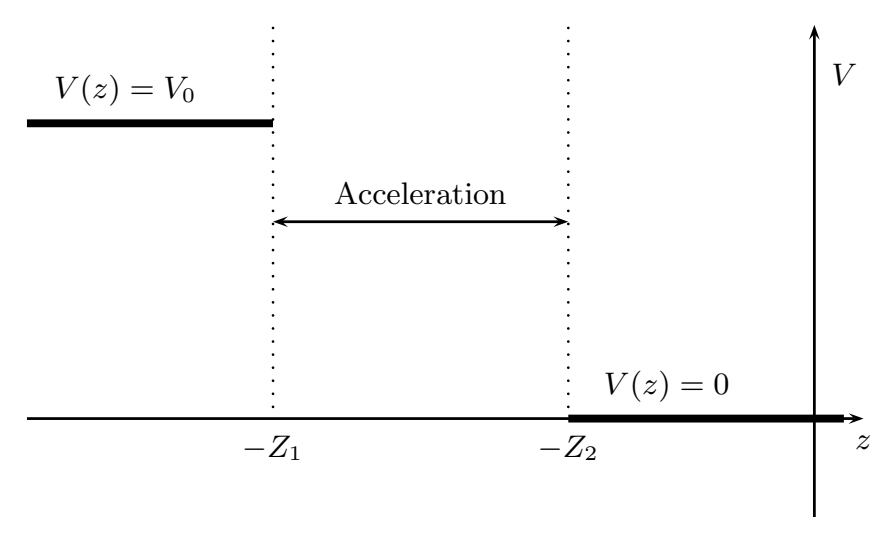

FIG. 2: The potential $V(z)$ and period of acceleration

for $z<-Z_{1}$ and $V(z)=0$ for $-Z_{2}<z$ for some $Z_{1}$ and $Z_{2}$, both positive constants with $Z_{1}>Z_{2}$. Thus, there is non-zero acceleration only in the interval $\left(-Z_{1},-Z_{2}\right)$ as represented in Fig. 2 for the case with $V_{0}>0$.

The external 4-force in Eq. (1.1) representing the electric force corresponding to this potential is given as

$$
\begin{aligned}
& F_{\mathrm{ext}}^{t}=-V^{\prime}(z) d z / d \tau, \\
& F_{\mathrm{ext}}^{z}=-V^{\prime}(z) d t / d \tau, \\
& F_{\mathrm{ext}}^{x}=F_{\mathrm{ext}}^{y}=0 .
\end{aligned}
$$

The Lorentz-Dirac 4-force can be given in the following form:

$$
\begin{aligned}
& F_{\mathrm{LD}}^{t}=F_{\mathrm{LD}} d z / d \tau, \\
& F_{\mathrm{LD}}^{z}=F_{\mathrm{LD}} d t / d \tau, \\
& F_{\mathrm{LD}}^{x}=F_{\mathrm{LD}}^{y}=0
\end{aligned}
$$

with

$$
F_{\mathrm{LD}} \equiv \frac{2 \alpha_{c}}{3} \gamma \frac{d}{d t}\left(\gamma^{3} \ddot{z}\right) .
$$

A dot indicates the derivative with respect to $t$. We have defined $\gamma \equiv\left(1-\dot{z}^{2}\right)^{-1 / 2}$ as usual.

Suppose that this particle would be at $z=0$ at time $t=0$ if the Lorentz-Dirac force was absent. The true position at $t=0$, which we denote $\delta z$ and call the position shift, can readily be found to lowest non-trivial order in $F_{\mathrm{LD}}$ by treating the Lorentz-Dirac force as perturbation. The calculation can be facilitated by using the fact that the total energy, $m d t / d \tau+V(z)$, changes by the amount of work done by the Lorentz-Dirac force. Thus we find

$$
\begin{aligned}
\int_{-\infty}^{t} F_{\mathrm{LD}}\left(t^{\prime}\right) \dot{z}\left(t^{\prime}\right) d t^{\prime} & =\frac{d}{d \dot{z}} \frac{m}{\sqrt{1-\dot{z}^{2}}} \delta \dot{z}+V^{\prime}(z) \delta z \\
& =m \gamma^{3} \dot{z}^{2} \frac{d}{d t}\left(\frac{\delta z}{\dot{z}}\right)
\end{aligned}
$$

where we have used

$$
\frac{d}{d t}(m \gamma \dot{z})=m \gamma^{3} \ddot{z}=-V^{\prime}(z)
$$


to zeroth order, i.e. in the absence of the Lorentz-Dirac force. Rearranging and integrating, and then interchanging the order of integration, we obtain the position shift as

$$
\delta z_{\mathrm{LD}}=-\frac{v_{0}}{m} \int_{-\infty}^{0}\left(\int_{0}^{t} \frac{1}{\gamma^{3}\left(t^{\prime}\right)\left[\dot{z}\left(t^{\prime}\right)\right]^{2}} d t^{\prime}\right) F_{\mathrm{LD}} \frac{d z}{d t} d t
$$

where $v_{0}=\left.\dot{z}\right|_{t=0}$ is the final velocity in the absence of radiation reaction. We shall see that this result is reproduced in QED in the $\hbar \rightarrow 0$ limit.

It is useful for later purposes to find the change in the position shift (2.6) caused by letting the particle be at $z=z_{0} \neq 0$ at $t=0$ with the same final velocity $v_{0}$ in the absence of the Lorentz-Dirac force. (We assume that $z_{0}>-Z_{2}$.) The time this particle spends after the acceleration and before getting to $t=0$ (when the position shift is "measured") is lengthened by $z_{0} / v_{0}$. Hence this position shift is obtained by using the same trajectory but defining it at $t_{0} \equiv z_{0} / v_{0}$. Hence, writing this position shift as

$$
\delta z_{\text {class }}=\delta z_{\mathrm{LD}}+\delta z_{\text {extra }}
$$

we find

$$
\delta z_{\text {extra }}=-\frac{v_{0}}{m} \int_{-\infty}^{t_{0}}\left(\int_{t_{0}}^{0} \frac{1}{\gamma^{3}\left(t^{\prime}\right)\left[\dot{z}\left(t^{\prime}\right)\right]^{2}} d t^{\prime}\right) F_{\mathrm{LD}} \frac{d z}{d t} d t
$$

Noting that $\dot{z}(t)=v_{0}$ for values of $t$ between 0 and $t_{0}$ we obtain

$$
\delta z_{\text {extra }}=-\frac{z_{0}}{m \gamma_{0}^{3} v_{0}^{2}} E_{\mathrm{em}}
$$

with $\gamma_{0} \equiv\left(1-v_{0}^{2}\right)^{-1 / 2}$, where $E_{\text {em }}$ is the energy emitted as radiation given by

$$
\begin{aligned}
E_{\mathrm{em}} & =-\int_{-\infty}^{0} F_{\mathrm{LD}} \frac{d z}{d t} d t \\
& =\frac{2 \alpha_{c}}{3} \int_{-\infty}^{0}\left(\gamma^{3} \ddot{z}\right)^{2} d t
\end{aligned}
$$

which is the relativistic Larmor formula for onedimensional motion. We shall see that the extra contribution (2.9) to the position shift is also reproduced by QED in the $\hbar \rightarrow 0$ limit.

\section{THE QED MODEL}

We now turn our attention to the quantum field theoretic model. The corresponding Lagrangian density is

$$
\begin{aligned}
\mathcal{L}= & {\left[\left(D_{\mu}+i e A_{\mu}\right) \varphi\right]^{\dagger}\left[\left(D^{\mu}+i e A^{\mu}\right) \varphi\right]-(m / \hbar)^{2} \varphi^{\dagger} \varphi } \\
& -\frac{1}{4} F_{\mu \nu} F^{\mu \nu}-\frac{1}{2}\left(\partial_{\mu} A^{\mu}\right)^{2}
\end{aligned}
$$

where $F_{\mu \nu} \equiv \partial_{\mu} A_{\nu}-\partial_{\nu} A_{\mu}$ and $D_{\mu} \equiv \partial_{\mu}+i V_{\mu} / \hbar$. The field $\varphi$ describes a charged scalar particle with mass $m$ and charge $e$, the field $A_{\mu}$ is the electromagnetic field, and the function $V_{\mu}$ is the external potential which accelerates the charged scalar particle and is given as $V_{\mu}=V(z) \delta_{\mu 0}$ for the static potential. The non-interacting quantum electromagnetic field $A_{\mu}(x)$ is expanded as

$$
A_{\mu}(x)=\int \frac{d^{3} \mathbf{k}}{2 k(2 \pi)^{3}}\left[a_{\mu}(\mathbf{k}) e^{-i k \cdot x}+a_{\mu}^{\dagger}(\mathbf{k}) e^{i k \cdot x}\right],
$$

where $k=\|\mathbf{k}\|$ and the annihilation and creation operators, $a_{\mu}(\mathbf{k})$ and $a_{\mu}^{\dagger}(\mathbf{k})$, respectively, for the photons with momenta $\hbar \mathbf{k}$ in the Feynman gauge satisfy

$$
\left[a_{\mu}(\mathbf{k}), a_{\nu}^{\dagger}\left(\mathbf{k}^{\prime}\right)\right]=-g_{\mu \nu}(2 \pi)^{3} 2 \hbar k \delta\left(\mathbf{k}-\mathbf{k}^{\prime}\right) .
$$

The Fourier expansion of the non-interacting quantum scalar field is not as straightforward because its field equation involves the potential $V(z)$ as follows:

$$
\left\{\hbar^{2}\left[i \partial_{t}-V(z)\right]^{2}+\hbar^{2} \nabla^{2}-m^{2}\right\} \varphi=0 .
$$

Positive-frequency mode functions can be found in the form

$$
\Phi_{\mathbf{p}}(t, \mathbf{x})=\phi_{p}(z) \exp \left[\frac{i}{\hbar}\left(\mathbf{p}_{\perp} \cdot \mathbf{x}_{\perp}-p_{0} t\right)\right], \quad p_{0}>0,
$$

where $\mathbf{x}_{\perp} \equiv(x, y)$ and $\mathbf{p}_{\perp} \equiv\left(p^{x}, p^{y}\right)$. We define the $z$-component of the momentum $p \equiv p^{z}$ by the relation $p_{0}^{2}=p^{2}+\mathbf{p}_{\perp}^{2}+m^{2}$. We let $p$ be positive (negative) if the mode function corresponds to a wave coming in from $z=-\infty(z=+\infty)$. The function $\phi_{p}(z)$ with $\mathbf{p}_{\perp}=0$ satisfies

$$
-\hbar^{2} \frac{d^{2}}{d z^{2}} \phi_{p}(z)=\left[\kappa_{p}(z)\right]^{2} \phi_{p}(z)
$$

where

$$
\kappa_{p}(z) \equiv \sqrt{\left[p_{0}-V(z)\right]^{2}-m^{2}} .
$$

If $\mathbf{p}_{\perp} \neq 0$, then the mass term $m^{2}$ has to be replaced by $m^{2}+\mathbf{p}_{\perp}^{2}$. However, we need only the cases with $\mathbf{p}_{\perp}=0$ and $p>0$ in our calculations. The WKB approximation for the function $\phi_{p}(z)$ with $p>0$, which will be useful later, reads

$$
\phi_{p}(z)=\sqrt{\frac{p}{\kappa_{p}(z)}} \exp \left[\int_{0}^{z} \kappa_{p}(\zeta) d \zeta\right]
$$

where we have required $\phi_{p}(0)=1$.

The non-interacting quantum charged scalar field is expanded in terms of these mode functions as

$$
\varphi(x)=\hbar \int \frac{d^{3} \mathbf{p}}{2 p_{0}(2 \pi \hbar)^{3}}\left[A(\mathbf{p}) \Phi_{\mathbf{p}}(x)+B^{\dagger}(\mathbf{p}) \Phi_{\mathbf{p}}^{*}(x)\right] .
$$

(This expansion needs to be modified if there are modes which are totally reflected by the potential - this is the case if $V_{0} \neq 0$ - or if there are modes which fail to reach $|z|=\infty$. However, such modifications will not 
be relevant since the affected modes will not enter our calculations.) The operators $A(\mathbf{p})$ and $B(\mathbf{p})$ satisfy

$$
\left[A(\mathbf{p}), A^{\dagger}\left(\mathbf{p}^{\prime}\right)\right]=\left[B(\mathbf{p}), B^{\dagger}\left(\mathbf{p}^{\prime}\right)\right]=(2 \pi \hbar)^{3} 2 p_{0} \delta\left(\mathbf{p}-\mathbf{p}^{\prime}\right),
$$

if one normalizes the mode functions appropriately, with all other commutators vanishing. The WKB mode functions $\Phi_{\mathbf{p}}(t, \mathbf{x})$ with $\phi_{p}(z)$ given by Eq. (3.8) are correctly normalized since it satisfies the usual normalization condition $\left|\Phi_{\mathbf{p}}(t, \mathbf{x})\right|=1$ in the region $\left(-Z_{2},+\infty\right)$ where $V(z)=0$.

Now, in the interaction picture, if there is one scalar particle with momentum $\mathbf{p}$ in the initial state, then the final state to lowest non-trivial order in $e$ is a superposition of a state proportional to the initial state and states with one scalar particle and one photon. Thus, the state $A^{\dagger}(\mathbf{p})|0\rangle$ evolves as follows:

$$
\begin{aligned}
A^{\dagger}(\mathbf{p})|0\rangle \rightarrow & {[1+i \mathcal{F}(\mathbf{p})] A^{\dagger}(\mathbf{p})|0\rangle } \\
& +\frac{i}{\hbar} \int \frac{d^{3} \mathbf{k}}{2 k(2 \pi)^{3}} \mathcal{A}^{\mu}(\mathbf{p}, \mathbf{k}) a_{\mu}^{\dagger}(\mathbf{k}) A^{\dagger}(\mathbf{P})|0\rangle .
\end{aligned}
$$

The $\mathcal{F}(\mathbf{p})$ is the forward-scattering amplitude of order $e^{2}$ coming from the one-loop diagram, which we do not evaluate explicitly. The emission amplitude $\mathcal{A}_{\mu}(\mathbf{p}, \mathbf{k})$ will play a central role in our calculations. Note that the momentum $\hbar \mathbf{k}$ of the photon is of order $\hbar$ because the wave number $\mathbf{k}$ rather than the momentum has the classical limit. The momentum $\mathbf{P}$ of the charged particle in the final state is determined using conservation of the transverse momentum and energy:

$$
\begin{aligned}
\mathbf{p}_{\perp} & =\mathbf{P}_{\perp}+\hbar \mathbf{k}_{\perp}, \\
p_{0} & =P_{0}+\hbar k .
\end{aligned}
$$

The $z$-component $P \equiv P^{z}$ is determined by the on-shell condition

$$
P_{0}^{2}=P^{2}+\mathbf{P}_{\perp}^{2}+m^{2} .
$$

We shall consider the initial state $|i\rangle$ given by

$$
|i\rangle=\int \frac{d^{3} \mathbf{p}}{\sqrt{2 p_{0}}(2 \pi \hbar)^{3}} f(\mathbf{p}) A^{\dagger}(\mathbf{p})|0\rangle,
$$

where the function $f(\mathbf{p})$ is sharply peaked about a momentum in the positive $z$-direction with width of order $\hbar$. We also require that the $p$-derivative of $f(\mathbf{p})$ has width of order $\hbar$. This wave packet corresponds to the classical particle considered in the previous section. We make a further assumption that the WKB approximation (3.8) is valid for the momenta $\mathbf{p}$ where the function $f(\mathbf{p})$ is not negligibly small. The WKB approximation is known to be applicable if the wavelength stays approximately constant over many periods (see, e.g. Ref. [15]). Since the "time-dependent wavelength" is $2 \pi \hbar\left[\kappa_{p}(z)\right]^{-1}$, where $\kappa_{p}(z)$ is defined by Eq. (3.7), this condition is

$$
\hbar\left|\frac{d}{d z}\left[\frac{1}{\kappa_{p}(z)}\right]\right| \ll 1,
$$

which is automatically satisfied as long as $\kappa_{p}(z)$ and $\kappa_{p}^{\prime}(z)$ can be regarded as quantities of order $\hbar^{0}$.

The normalization condition $\langle i \mid i\rangle=1$ implies

$$
\int \frac{d^{3} \mathbf{p}}{(2 \pi \hbar)^{3}}|f(\mathbf{p})|^{2}=1
$$

because of the commutation relations (3.10). This equation shows that the function $f(\mathbf{p})$ can heuristically be regarded as the one-particle wave function in the momentum representation. In Ref. 11] it was assumed that $f(\mathbf{p})$ was real for simplicity. This assumption will be dropped in this paper.

\section{POSITION EXPECTATION VALUE}

In this section we derive a formula for the position shift in terms of the emission amplitude $\mathcal{A}^{\mu}(\mathbf{p}, \mathbf{k})$. We closely follow Ref. 10], correcting a few errors. In particular, the fourth term in Eq. (4.32) was missing in Ref. 10].

\section{A. Non-radiating particle}

Define the charge density by

$$
\rho(x) \equiv \frac{i}{\hbar}: \varphi^{\dagger} \partial_{t} \varphi-\partial_{t} \varphi^{\dagger} \cdot \varphi:,
$$

where : . . : denotes normal ordering. The operator $\rho(x)$ is the $t$-component of the conserved current

$$
J^{\mu}(x) \equiv \frac{i}{\hbar}: \varphi^{\dagger} \partial^{\mu} \varphi-\partial^{\mu} \varphi^{\dagger} \cdot \varphi: .
$$

We let the potential satisfy $\left|V_{0}\right|<2 m$, thus precluding the possibility of scalar-particle pair creation. Then, the charge density $\rho(x)$ coincides with the probability density for the particle if there is only one charged particle in the state. Hence the expectation value of $z$ is

$$
\langle z\rangle=\int d^{3} \mathbf{x} z\langle\rho(t, \mathbf{x})\rangle
$$

We shall first need to consider the expectation value of $z$ for the hypothetical scalar particle that interact with the external potential but not with the quantum electromagnetic field. This will be the benchmark against which we can define the position shift due to the radiation. Hence, we must evaluate the expectation value of the probability density in the absence of radiation and use Eq. (4.3) to find $\langle z\rangle$. The initial and final states in this case are the same. Thus, using the commutation relations (3.10) for the expectation value of the charge density (4.1) in the state $|i\rangle$ given by (3.15), we find

$$
\begin{aligned}
& \langle\rho(t, \mathbf{x})\rangle \\
& =i \hbar \int \frac{d^{3} \mathbf{p}^{\prime}}{\sqrt{2 p_{0}^{\prime}}(2 \pi \hbar)^{3}} \int \frac{d^{3} \mathbf{p}}{\sqrt{2 p_{0}}(2 \pi \hbar)^{3}} f^{*}\left(\mathbf{p}^{\prime}\right) f(\mathbf{p}) \\
& \times\left[\Phi_{\mathbf{p}^{\prime}}^{*}(t, \mathbf{x}) \partial_{t} \Phi_{\mathbf{p}}(t, \mathbf{x})-\partial_{t} \Phi_{\mathbf{p}^{\prime}}^{*}(t, \mathbf{x}) \cdot \Phi_{\mathbf{p}}(t, \mathbf{x})\right] .
\end{aligned}
$$


The $t$-dependence of the mode functions is given by $\Phi_{\mathbf{p}}(t, \mathbf{x}) \propto e^{-i p_{0} t / \hbar}$. Hence, we find

$$
\begin{aligned}
\langle\rho(t, \mathbf{x})\rangle= & \frac{1}{2} \int \frac{d^{3} \mathbf{p}^{\prime}}{(2 \pi \hbar)^{3}} \int \frac{d^{3} \mathbf{p}}{(2 \pi \hbar)^{3}} f^{*}\left(\mathbf{p}^{\prime}\right) f(\mathbf{p}) \\
& \times\left(\sqrt{\frac{p_{0}}{p_{0}^{\prime}}}+\sqrt{\frac{p_{0}^{\prime}}{p_{0}}}\right) \Phi_{\mathbf{p}^{\prime}}^{*}(t, \mathbf{x}) \Phi_{\mathbf{p}}(t, \mathbf{x}) .
\end{aligned}
$$

We arrange the wave packet so that at time $t=0$ it is located far into the region where the potential vanishes. Then we may approximate the mode functions as follows:

$$
\Phi_{\mathbf{p}}(0, \mathbf{x}) \approx e^{-i\left(p z+\mathbf{p}_{\perp} \cdot \mathbf{x}_{\perp}\right) / \hbar} .
$$

After substituting Eq. 4.5) in the position expectation formula (4.3) we use the approximation (4.6) to write

$$
z \Phi_{\mathbf{p}}(0, \mathbf{x}) \approx-i \hbar \frac{\partial}{\partial p} \Phi_{\mathbf{p}}(0, \mathbf{x}) .
$$

We can now use integration by parts for the variable $p$ in Eq. (4.3) to obtain the expectation value of $z$ for the hypothetical non-radiating particle, which we denote by $z_{0}$, as

$$
\begin{aligned}
z_{0} & =\frac{i \hbar}{2} \int \frac{d^{3} \mathbf{p}^{\prime}}{(2 \pi \hbar)^{3}} \int \frac{d^{3} \mathbf{p}}{(2 \pi \hbar)^{3}} \\
& \times \frac{\partial}{\partial p}\left[\left(\sqrt{\frac{p_{0}}{p_{0}^{\prime}}}+\sqrt{\frac{p_{0}^{\prime}}{p_{0}}}\right) f^{*}\left(\mathbf{p}^{\prime}\right) f(\mathbf{p})\right] \\
& \times \int d^{3} \mathbf{x} \Phi_{\mathbf{p}^{\prime}}^{*}(0, \mathbf{x}) \Phi_{\mathbf{p}}(0, \mathbf{x}) .
\end{aligned}
$$

Substituting the approximation (4.6) for $\Phi_{\mathbf{p}}$ gives a delta function:

$$
\begin{aligned}
z_{0} & =\frac{i \hbar}{2} \int \frac{d^{3} \mathbf{p}^{\prime}}{(2 \pi \hbar)^{3}} \int \frac{d^{3} \mathbf{p}}{(2 \pi \hbar)^{3}} f^{*}\left(\mathbf{p}^{\prime}\right) \\
& \times \frac{\partial}{\partial p}\left[\left(\sqrt{\frac{p_{0}}{p_{0}^{\prime}}}+\sqrt{\frac{p_{0}^{\prime}}{p_{0}}}\right) f(\mathbf{p})\right] \\
& \times(2 \pi \hbar)^{3} \delta\left(\mathbf{p}-\mathbf{p}^{\prime}\right) .
\end{aligned}
$$

Performing the $\mathbf{p}^{\prime}$-integration after explicitly working out the $p$-derivative, we find

$$
z_{0}=i \hbar \int \frac{d^{3} \mathbf{p}}{(2 \pi \hbar)^{3}} f^{*}(\mathbf{p}) \frac{\partial}{\partial p} f(\mathbf{p})
$$

or, by integration by parts,

$$
z_{0}=\frac{i \hbar}{2} \int \frac{d^{3} \mathbf{p}}{(2 \pi \hbar)^{3}} f^{*}(\mathbf{p}) \stackrel{\leftrightarrow}{\partial}_{p} f(\mathbf{p})
$$

where $\overleftrightarrow{\partial}_{p}=\vec{\partial}_{p}-\overleftarrow{\partial}_{p}$. This result can be interpreted as the expectation value of the position operator $i \hbar \partial_{p}$ in the p-representation of the one-particle wave function. An example of a function $f(\mathbf{p})$ satisfying Eq. (4.11) is $f(\mathbf{p})=f_{R}(\mathbf{p}) e^{-i z_{0} p / \hbar}$ with the function $f_{R}(\mathbf{p})$ being real though it is not necessary to make this assumption.

\section{B. Radiating particle}

Now we consider the case of the radiating particle. The final state resulting from the initial state $|i\rangle$ can be found from Eq. (3.11) as

$$
\begin{aligned}
&|f\rangle= \int \frac{d^{3} \mathbf{p}}{\sqrt{2 p_{0}}(2 \pi \hbar)^{3}} F(\mathbf{p}) A^{\dagger}(\mathbf{p})|0\rangle \\
&+\frac{i}{\hbar} \int \frac{d^{3} \mathbf{k}}{2 k(2 \pi)^{3}} \int \frac{d^{3} \mathbf{p}}{\sqrt{2 p_{0}}(2 \pi \hbar)^{3}} \\
& \quad \times G^{\mu}(\mathbf{p}, \mathbf{k}) a_{\mu}^{\dagger}(\mathbf{k}) A^{\dagger}(\mathbf{P})|0\rangle
\end{aligned}
$$

where $\mathbf{P}_{\perp}=\mathbf{p}_{\perp}-\hbar \mathbf{k}_{\perp}$ and $P_{0}=p_{0}-\hbar k$ as before. We have defined

$$
\begin{gathered}
F(\mathbf{p}) \equiv[1+i \mathcal{F}(\mathbf{p})] f(\mathbf{p}) \\
G^{\mu}(\mathbf{p}, \mathbf{k}) \equiv \mathcal{A}^{\mu}(\mathbf{p}, \mathbf{k}) f(\mathbf{p})
\end{gathered}
$$

One can heuristically regard the function $F(\mathbf{p})$ as the one-particle wave function in the zero-photon sector in the p-representation and the function $G^{\mu}(\mathbf{p}, \mathbf{k})$ as that in the one-photon sector with a photon with momentum $\hbar \mathbf{k}$ in the $\mathbf{P}$-representation. By introducing the definition

$$
C^{\dagger \mu}(\mathbf{k})=\frac{i}{\hbar} \int \frac{d^{3} \mathbf{p}}{\sqrt{2 p_{0}}(2 \pi \hbar)^{3}} \mathcal{A}^{\mu}(\mathbf{p}, \mathbf{k}) f(\mathbf{p}) A^{\dagger}(\mathbf{P}),
$$

the final state can be written

$$
\begin{aligned}
|f\rangle= & \int \frac{d^{3} \mathbf{p}}{\sqrt{2 p_{0}}(2 \pi \hbar)^{3}} F(\mathbf{p}) A^{\dagger}(\mathbf{p})|0\rangle \\
& +\int \frac{d^{3} \mathbf{k}}{2 k(2 \pi)^{3}} C^{\dagger \mu}(\mathbf{k}) a_{\mu}^{\dagger}(\mathbf{k})|0\rangle .
\end{aligned}
$$

We first derive the relation between the imaginary part of $\mathcal{F}(\mathbf{p})$ and the scattering probability which results from unitarity. Recalling the definition (4.13) and using the unitarity of time evolution, we find

$$
1=\langle f \mid f\rangle=\int \frac{d^{3} \mathbf{p}}{(2 \pi)^{3}}(1-2 \operatorname{Im} \mathcal{F}(\mathbf{p}))|f(\mathbf{p})|^{2}+\mathcal{P}_{\mathrm{em}},
$$

to first order in $e^{2}$, where the emission probability $\mathcal{P}_{\text {em }}$ is given by

$$
\begin{aligned}
\mathcal{P}_{\mathrm{em}}= & -\hbar \int \frac{d^{3} \mathbf{k}}{2 k(2 \pi)^{3}}\left\langle 0\left|C_{\mu}\left(\mathbf{k}^{\prime}\right) C^{\dagger \mu}(\mathbf{k})\right| 0\right\rangle \\
= & -\frac{1}{\hbar} \int \frac{d^{3} \mathbf{k}}{2 k(2 \pi)^{3}} \int \frac{d^{3} \mathbf{p}}{\sqrt{2 p_{0}}(2 \pi \hbar)^{3}} f^{*}(\mathbf{p}) \mathcal{A}_{\mu}^{*}(\mathbf{p}, \mathbf{k}) \\
& \times \int \frac{d^{3} \mathbf{p}^{\prime}}{\sqrt{2 p_{0}^{\prime}}(2 \pi \hbar)^{3}} f\left(\mathbf{p}^{\prime}\right) \mathcal{A}^{\mu}\left(\mathbf{p}^{\prime}, \mathbf{k}\right) \\
& \times\left\langle 0\left|A(\mathbf{P}) A^{\dagger}\left(\mathbf{P}^{\prime}\right)\right| 0\right\rangle
\end{aligned}
$$

To perform the $\mathbf{p}^{\prime}$-integration recall first that

$$
\left\langle 0\left|A(\mathbf{P}) A^{\dagger}\left(\mathbf{P}^{\prime}\right)\right| 0\right\rangle=(2 \pi \hbar)^{3} 2 P_{0} \delta\left(\mathbf{P}-\mathbf{P}^{\prime}\right) .
$$


We need to integrate this delta function with respect to $\mathbf{p}^{\prime}$ by relating it to $\delta\left(\mathbf{p}-\mathbf{p}^{\prime}\right)$. We note that

$$
\delta\left(\mathbf{P}_{\perp}-\mathbf{P}_{\perp}^{\prime}\right)=\delta\left(\mathbf{p}_{\perp}-\mathbf{p}_{\perp}^{\prime}\right)
$$

because $\mathbf{P}_{\perp}=\mathbf{p}_{\perp}-\hbar \mathbf{k}_{\perp}$, and

$$
\delta\left(P-P^{\prime}\right)=\frac{d p}{d P} \delta\left(p-p^{\prime}\right) .
$$

By using these formulae in Eq. (4.19) we find

$$
\left\langle 0\left|A(\mathbf{P}) A^{\dagger}\left(\mathbf{P}^{\prime}\right)\right| 0\right\rangle=\frac{P_{0}}{p_{0}} \frac{d p}{d P}(2 \pi \hbar)^{3} 2 p_{0} \delta\left(\mathbf{p}-\mathbf{p}^{\prime}\right) .
$$

By substituting this formula in Eq. (4.18) we obtain

$$
\begin{aligned}
\mathcal{P}_{\mathrm{em}}= & -\frac{1}{\hbar} \int \frac{d^{3} \mathbf{p}}{(2 \pi \hbar)^{3}}|f(\mathbf{p})|^{2} \\
& \times \int \frac{d^{3} \mathbf{k}}{2 k(2 \pi)^{3}} \mathcal{A}_{\mu}^{*}(\mathbf{p}, \mathbf{k}) \mathcal{A}^{\mu}\left(\mathbf{p}^{\prime}, \mathbf{k}\right) \frac{P_{0}}{p_{0}} \frac{d p}{d P} .
\end{aligned}
$$

Eq. (4.17) must hold for any function $f(\mathbf{p})$, so we must have

$$
2 \operatorname{Im} \mathcal{F}(\mathbf{p})=-\frac{1}{\hbar} \int \frac{d^{3} \mathbf{k}}{2 k(2 \pi)^{3}} \mathcal{A}_{\mu}^{*}(\mathbf{p}, \mathbf{k}) \mathcal{A}^{\mu}(\mathbf{p}, \mathbf{k}) \frac{P_{0}}{p_{0}} \frac{d p}{d P} .
$$

The contribution of the one-photon-emission term to $\langle\rho(t, \mathbf{x})\rangle$ is

$$
\langle\rho(t, \mathbf{x})\rangle_{\mathrm{em}}=-\hbar \int \frac{d^{3} \mathbf{k}}{2 k(2 \pi)^{3}}\left\langle 0\left|C^{\mu}(\mathbf{k}) \rho(t, \mathbf{x}) C_{\mu}^{\dagger}(\mathbf{k})\right| 0\right\rangle .
$$

The integrand here is obtained from $\langle\rho(t, \mathbf{x})\rangle$ for the nonradiating particle if we replace the state $|i\rangle$ in Eq. (3.15) by

$$
\hbar^{1 / 2} C_{\mu}^{\dagger}(\mathbf{k})|0\rangle=i \int \frac{d^{3} \mathbf{P}}{\sqrt{2 P_{0}}(2 \pi \hbar)^{3}} g_{\mu}(\mathbf{P}) A^{\dagger}(\mathbf{P})|0\rangle,
$$

where

$$
g_{\mu}(\mathbf{P}) \equiv \hbar^{-1 / 2} \mathcal{A}_{\mu}(\mathbf{p}, \mathbf{k}) f(\mathbf{p}) \sqrt{\frac{P_{0}}{p_{0}}} \frac{d p}{d P} .
$$

Hence we conclude from Eq. (4.11) that

$$
\begin{aligned}
& \hbar \int d^{3} \mathbf{x} z\left\langle 0\left|C^{\mu}(\mathbf{k}) \rho(t, \mathbf{x}) C_{\mu}^{\dagger}(\mathbf{k})\right| 0\right\rangle \\
& =\frac{i \hbar}{2} \int \frac{d^{3} \mathbf{P}}{(2 \pi)^{3}} g^{\mu *}(\mathbf{P}) \stackrel{\leftrightarrow}{\partial_{P}} g_{\mu}(\mathbf{P}) .
\end{aligned}
$$

This implies that the one-photon-emission contribution to $\langle z\rangle$ is

$$
\begin{aligned}
\langle z\rangle_{\mathrm{em}}= & -\frac{i}{2} \int \frac{d^{3} \mathbf{k}}{2 k(2 \pi)^{3}} \int \frac{d^{3} \mathbf{P}}{(2 \pi \hbar)^{3}} \\
& \times\left[f^{*}(\mathbf{p}) \mathcal{A}^{\mu *}(\mathbf{p}, \mathbf{k})\right] \stackrel{\leftrightarrow}{\partial}_{P}\left[f(\mathbf{p}) \mathcal{A}_{\mu}(\mathbf{p}, \mathbf{k})\right] \\
& \times \frac{P_{0}}{p_{0}}\left(\frac{d p}{d P}\right)^{2} .
\end{aligned}
$$

We convert the integration variable from $\mathbf{P}$ to $\mathbf{p}$ by using $d^{3} \mathbf{P}=(\partial P / \partial p) d^{3} \mathbf{p}$ and the $P$-derivative to a $p$-derivative by using $\partial_{P}=(\partial p / \partial P) \partial_{p}$ to find

$$
\begin{aligned}
\langle z\rangle_{\mathrm{em}}= & -\frac{i}{2} \int \frac{d^{3} \mathbf{p}}{(2 \pi \hbar)^{3}} f^{*}(\mathbf{p}) \stackrel{\leftrightarrow}{\partial}_{p} f(\mathbf{p}) \\
& \times \int \frac{d^{3} \mathbf{k}}{2 k(2 \pi)^{3}} \mathcal{A}^{\mu *}(\mathbf{p}, \mathbf{k}) \mathcal{A}_{\mu}(\mathbf{p}, \mathbf{k}) \frac{P_{0}}{p_{0}}\left(\frac{d p}{d P}\right)^{2} \\
& -\frac{i}{2} \int \frac{d^{3} \mathbf{p}}{(2 \pi \hbar)^{3}}|f(\mathbf{p})|^{2} \\
& \times \int \frac{d^{3} \mathbf{k}}{2 k(2 \pi)^{3}} \mathcal{A}^{\mu *}(\mathbf{p}, \mathbf{k}) \stackrel{\leftrightarrow}{\partial}_{p} \mathcal{A}_{\mu}(\mathbf{p}, \mathbf{k}) .
\end{aligned}
$$

We have dropped the factor $\left(P_{0} / p_{0}\right)(d p / d P)^{2}$ from the second term for the following reason. It will turn out later that the emission amplitude $\mathcal{A}^{\mu}$ and its $p$-derivative are both of order $\hbar^{0}$. Thus the second term in the above expression for $\langle z\rangle_{\mathrm{em}}$ is of order $\hbar^{0}$. Since $P_{0}=p_{0}$ and $d p / d P=1$ to order $\hbar^{0}$, we can drop the factors of $P_{0} / p_{0}$ and $d p / d P$ from the second term. However, one needs to keep these factors in the first term, which is of order $\hbar^{-1}$.

From the expressions for the final state (4.16) it is clear that the contribution to $\langle z\rangle$ from the process without photon emission is obtained from Eq. (4.11) by replacing $f(\mathbf{p})$ with $F(\mathbf{p}) \equiv[1+i \mathcal{F}(\mathbf{p})] f(\mathbf{p})$. Thus

$$
\begin{aligned}
\langle z\rangle_{\text {for }}= & \frac{i \hbar}{2} \int \frac{d^{3} \mathbf{p}}{(2 \pi \hbar)^{3}}\left\{2 i|f(\mathbf{p})|^{2} \partial_{p} \operatorname{Re} \mathcal{F}(\mathbf{p})\right. \\
& \left.+[1-2 \operatorname{Im} \mathcal{F}(\mathbf{p})] f^{*}(\mathbf{p}) \stackrel{\leftrightarrow}{\partial}_{p} f(\mathbf{p})\right\}
\end{aligned}
$$

to first order in $e^{2}$. (Recall that the forward-scattering amplitude $\mathcal{F}(\mathbf{p})$ is of order $e^{2}$.) We can now add the two contributions $\langle z\rangle_{\mathrm{em}}$ and $\langle z\rangle_{\text {for }}$ together to find the position expectation value:

$$
\begin{aligned}
\langle z\rangle= & \frac{i \hbar}{2} \int \frac{d^{3} \mathbf{p}}{(2 \pi \hbar)^{3}} f^{*}(\mathbf{p}) \stackrel{\leftrightarrow}{\partial}_{p} f(\mathbf{p}) \\
- & \hbar \int \frac{d^{3} \mathbf{p}}{(2 \pi \hbar)^{3}}|f(\mathbf{p})|^{2} \partial_{p} \operatorname{Re} \mathcal{F}(\mathbf{p}) \\
- & \frac{i}{2} \int \frac{d^{3} \mathbf{p}}{(2 \pi \hbar)^{3}}|f(\mathbf{p})|^{2} \\
& \times \int \frac{d^{3} \mathbf{k}}{2 k(2 \pi)^{3}} \mathcal{A}^{\mu *}(\mathbf{p}, \mathbf{k}) \stackrel{\leftrightarrow}{\partial}_{p} \mathcal{A}_{\mu}(\mathbf{p}, \mathbf{k}) \\
- & \frac{i}{2} \int \frac{d^{3} \mathbf{p}}{(2 \pi \hbar)^{3}} f^{*}(\mathbf{p}) \stackrel{\leftrightarrow}{\partial}_{p} f(\mathbf{p}) \\
& \times \int \frac{d^{3} \mathbf{k}}{2 k(2 \pi)^{3}} \mathcal{A}^{\mu *}(\mathbf{p}, \mathbf{k}) \mathcal{A}_{\mu}(\mathbf{p}, \mathbf{k}) \\
& \times \frac{P_{0}}{p_{0}} \frac{d p}{d P}\left(\frac{d p}{d P}-1\right) .
\end{aligned}
$$

The unitarity relation (4.24) has been used to eliminate $2 \operatorname{Im} \mathcal{F}(\mathbf{p})$. Each term in Eq. (4.32) can be interpreted 
as follows. The first term is the position expectation value $z_{0}$ for the non-radiating particle. The second term is the contribution from what can be regarded as the one-loop quantum correction to the potential. Therefore, we identify the sum of the third and forth terms as the position shift to be compared with the classical position shift $\delta z_{\text {class }}$ given by Eq. (2.7). Using Eq. (4.11) and the assumption that the function $f(\mathbf{p})$ is sharply peaked about a momentum in the positive $z$-direction with the width of order $\hbar$, we find these two terms in the $\hbar \rightarrow 0$ limit as

$$
\delta z_{q}=\delta z_{q 1}+\delta z_{q 2}
$$

where

$$
\begin{aligned}
\delta z_{q 1}= & -\frac{i}{2} \int \frac{d^{3} \mathbf{k}}{2 k(2 \pi)^{3}} \\
& \times \mathcal{A}^{\mu *}(\mathbf{p}, \mathbf{k}) \stackrel{\leftrightarrow}{\partial}_{p} \mathcal{A}_{\mu}(\mathbf{p}, \mathbf{k}) \\
\delta z_{q 2}= & -\frac{z_{0}}{\hbar} \int \frac{d^{3} \mathbf{k}}{2 k(2 \pi)^{3}} \\
& \times \mathcal{A}^{\mu *}(\mathbf{p}, \mathbf{k}) \mathcal{A}_{\mu}(\mathbf{p}, \mathbf{k})\left(\frac{d p}{d P}-1\right)
\end{aligned}
$$

where the momentum at which the function $f(\mathbf{p})$ is peaked is now denoted simply by $\mathbf{p}$. We have used the fact that $d p / d P-1$ is of order $\hbar$ to drop the factor $\left(P_{0} / p_{0}\right)(d p / d P)$ in $\delta z_{q 2}$. We shall demonstrate that the quantum position shift $\delta z_{q}$ in the $\hbar \rightarrow 0$ limit is identical with the classical counterpart $\delta z_{\text {class }}$ by showing $\delta z_{q 1}=\delta z_{\mathrm{LD}}$ and $\delta z_{q 2}=\delta z_{\text {extra }}$.

Let us first examine the latter equality. To this end we need to find an expression for $d p / d P$ in terms of $p$ and $k$. The energy conservation equation $p_{0}-P_{0}=\hbar k$ gives a one-to-one relation between $p$ and $P$ for a given $k$ after letting $\mathbf{P}_{\perp}^{2}=\mathbf{p}_{\perp}^{2}=0$ because these are of order $\hbar^{2}$. Then we find

$$
\frac{d p}{d P}=1-\frac{m^{2}}{p^{2} p_{0}} \hbar k
$$

By using this formula in Eq. 4.35) we obtain

$$
\delta z_{q 2}=-\frac{m^{2} z_{0}}{p^{2} p_{0}} \mathcal{E}_{\mathrm{em}}
$$

where

$$
\mathcal{E}_{\mathrm{em}} \equiv-\int \frac{d^{3} \mathbf{k}}{2 k(2 \pi)^{3}} k \mathcal{A}^{\mu *}(\mathbf{p}, \mathbf{k}) \mathcal{A}_{\mu}(\mathbf{p}, \mathbf{k})
$$

is the expectation value of the energy emitted as radiation. By comparing Eq. (4.37) with Eq. (2.9), it can be seen that the equality $\delta z_{q 2}=\delta z_{\text {extra }}$ will hold if

$$
\mathcal{E}_{\mathrm{em}}=\frac{2 \alpha_{c}}{3} \int_{-\infty}^{0}\left(\gamma^{3} \ddot{z}\right)^{2} d t
$$

which is identical to the relativistic generalization of the classical Larmor formula. To show this equality (and also $\left.\delta z_{q 1}=\delta z_{\mathrm{LD}}\right)$ we need to find the $\hbar \rightarrow 0$ limit of the one-photon-emission amplitude $\mathcal{A}_{\mu}(\mathbf{p}, \mathbf{k})$ to which we now turn.

\section{EMISSION AMPLITUDE}

The one-photon-emission part of the evolution of the state was represented earlier in Eq. (3.11) by

$$
A^{\dagger}(\mathbf{p})|0\rangle \rightarrow \cdots+\frac{i}{\hbar} \int \frac{d^{3} \mathbf{k}}{2 k(2 \pi)^{3}} \mathcal{A}^{\mu}(\mathbf{p}, \mathbf{k}) a_{\mu}^{\dagger}(\mathbf{k}) A^{\dagger}(\mathbf{P})|0\rangle .
$$

The evolution in perturbation theory in the interaction picture is generated by the interaction Lagrangian density as follows:

$$
A^{\dagger}(\mathbf{p})|0\rangle \rightarrow \cdots+\frac{i}{\hbar} \int d^{4} x \mathcal{L}_{I}(x) A^{\dagger}(\mathbf{p})|0\rangle .
$$

We can take the inner product of the two expressions (5.1) and (5.2) with the state $\langle 0| a_{\nu}(\mathbf{k}) A\left(\mathbf{p}^{\prime}\right)$ and equate them in order to find the amplitude $\mathcal{A}^{\mu}(\mathbf{p}, \mathbf{k})$. This procedure leads to

$$
\begin{aligned}
& -2 \hbar p_{0}^{\prime}(2 \pi \hbar)^{3} \delta\left(\mathbf{p}^{\prime}-\mathbf{P}\right) \mathcal{A}_{\nu}(\mathbf{p}, \mathbf{k}) \\
& =\int d^{4} x\left\langle 0\left|a_{\nu}(\mathbf{k}) A\left(\mathbf{p}^{\prime}\right) \mathcal{L}_{I}(x) A^{\dagger}(\mathbf{p})\right| 0\right\rangle .
\end{aligned}
$$

Integrating over the variable $\mathbf{p}^{\prime}$, with the appropriate measure, and rearranging, we find that $\mathcal{A}_{\mu}(\mathbf{p}, \mathbf{k})$ is given by

$$
\begin{aligned}
\mathcal{A}_{\mu}(\mathbf{p}, \mathbf{k})=- & \frac{1}{\hbar} \int \frac{d^{3} \mathbf{p}^{\prime}}{2 p_{0}^{\prime}(2 \pi \hbar)^{3}} \\
& \times \int d^{4} x\left\langle 0\left|a_{\mu}(\mathbf{k}) A\left(\mathbf{p}^{\prime}\right) \mathcal{L}_{I}(x) A^{\dagger}(\mathbf{p})\right| 0\right\rangle
\end{aligned}
$$

The $\mathcal{L}_{I}(x)$ is obtained from the Lagrangian density 3.1 as follows:

$$
\mathcal{L}_{I}(x)=-\frac{i e}{\hbar} A_{\mu}:\left[\varphi^{\dagger} D^{\mu} \varphi-\left(D^{\mu} \varphi\right)^{\dagger} \varphi\right]: .
$$

Recall that $D_{\mu}=\partial_{\mu}+i V_{\mu}$, where $V_{\mu}=\delta_{\mu 0} V(z)$. Вy substituting Eq. (5.5) in Eq. (5.4) and using the commutation relations (3.3) and (3.10) we find

$$
\begin{aligned}
\mathcal{A}_{\mu}(\mathbf{p}, \mathbf{k}) & =-i e \hbar \int \frac{d^{3} \mathbf{p}^{\prime}}{2 p_{0}^{\prime}(2 \pi \hbar)^{3}} \int d^{4} x e^{i k \cdot x} \\
& \times\left[\Phi_{\mathbf{p}^{\prime}}^{*}(x) D_{\mu} \Phi_{\mathbf{p}}(x)-\left(D_{\mu} \Phi_{\mathbf{p}^{\prime}}(x)\right)^{\dagger} \Phi_{\mathbf{p}}(x)\right],
\end{aligned}
$$

where the mode functions $\Phi_{\mathbf{p}}(x)$ are given by Eq. (3.5). As we mentioned before, we assume that the WKB approximation (3.8) is valid for the initial mode function. Since the momentum $\hbar \mathbf{k}$ of the emitted photon is of order $\hbar$, the WKB approximation can also be used for the mode functions for the final state.

Since the transverse momentum $\mathbf{p}_{\perp}$ is assumed to be of order $\hbar$, the final transverse momentum $\mathbf{p}_{\perp}-\hbar \mathbf{k}_{\perp}$ is also of order $\hbar$. This means that we do not need to 
consider the $x$ - and $y$-components $\mathcal{A}_{\mu}(\mathbf{p}, \mathbf{k})$ because they are smaller by a factor of $\hbar$ compared to the $t$ - and $z$ components. Using $D_{0}=\partial_{t}+i V(z) / \hbar$ we obtain

$$
\begin{aligned}
\mathcal{A}_{t}(\mathbf{p}, \mathbf{k}) & =-e \int \frac{d^{3} \mathbf{p}^{\prime}}{2 p_{0}^{\prime}(2 \pi \hbar)^{3}} \int d^{4} x \\
& \times \phi_{p^{\prime}}^{*}(z) \phi_{p}(z)\left[p_{0}+p_{0}^{\prime}-2 V(z)\right] \\
& \times e^{i k \cdot x} e^{-i\left[\left(p_{0}^{\prime}-p_{0}\right) t-\left(\mathbf{p}_{\perp}^{\prime}-\mathbf{p}_{\perp}\right) \cdot \mathbf{x}_{\perp}\right] / \hbar}
\end{aligned}
$$

Integration with respect to $t, x$ and $y$ gives

$$
\begin{aligned}
\mathcal{A}_{t}(\mathbf{p}, \mathbf{k})= & -e \int \frac{d p^{\prime}}{2 p_{0}^{\prime}} \int d z \\
& \times\left[p_{0}+p_{0}^{\prime}-2 V(z)\right] \phi_{p^{\prime}}^{*}(z) \phi_{p}(z) e^{-i k^{z} z} \\
& \times \delta\left(p_{0}^{\prime}+\hbar k-p_{0}\right) .
\end{aligned}
$$

We convert the $p^{\prime}$-integration to $p_{0}^{\prime}$-integration by noting that $d p^{\prime} / p_{0}^{\prime}=d p_{0}^{\prime} / p^{\prime}$ and find

$$
\mathcal{A}_{t}(\mathbf{p}, \mathbf{k})=-e \int d z \frac{P_{0}+p_{0}-V(z)}{2 P} \phi_{P}^{*}(z) \phi_{p}(z) e^{-i k^{z} z}
$$

where $P_{0}=p_{0}-\hbar k$ with $P \equiv \sqrt{P_{0}^{2}-m^{2}}$ as before. We let $P_{0}=p_{0}$ and $P=p$ in Eq. (5.9) because the differences $P_{0}-p_{0}$ and $P-p$ are of order $\hbar$. Thus, we obtain

$$
\mathcal{A}_{t}(\mathbf{p}, \mathbf{k})=-e \int d z \frac{p_{0}-V(z)}{p} \phi_{P}^{*}(z) \phi_{p}(z) e^{-i k^{z} z}
$$

Proceeding similarly for the $z$-component $\mathcal{A}_{z}(\mathbf{p}, \mathbf{k})$ we have

$$
\begin{aligned}
\mathcal{A}_{z}(\mathbf{p}, \mathbf{k})= & -\frac{i e \hbar}{2 p} \int d z \\
& \times\left[\phi_{P}^{*}(z) \frac{d \phi_{p}(z)}{d z}-\frac{d \phi_{P}^{*}(z)}{d z} \phi_{p}(z)\right] e^{-i k^{z} z} .
\end{aligned}
$$

To simplify the expression for the emission amplitude $\mathcal{A}_{\mu}$ further, we need to use the explicit form of $\phi_{p}(z)$ given by Eq. (3.8). We note first

$$
\begin{aligned}
\phi_{P}^{*}(z) \phi_{p}(z)= & \sqrt{\frac{P p}{\kappa_{P}(z) \kappa_{p}(z)}} \\
& \times \exp \left\{\frac{i}{\hbar} \int_{0}^{z}\left[\kappa_{p}(\eta)-\kappa_{P}(\eta)\right] d \eta\right\} .
\end{aligned}
$$

Now, to lowest order in $\hbar$ we have

$$
\kappa_{p}(z)-\kappa_{P}(z)=\frac{\partial \kappa_{p}(z)}{\partial p_{0}}\left(p_{0}-P_{0}\right)=\frac{p_{0}-V(z)}{\kappa_{p}(z)} \hbar k .
$$

Note that $\kappa_{p}(z)$ and $p_{0}-V(z)$ are the $z$ - and $t$ components of $m d x^{\mu} / d \tau$, respectively, of the classical particle with final momentum $p$ and vanishing transverse momentum. Hence, $v_{p}(z) \equiv \kappa_{p}(z) /\left[p_{0}-V(z)\right]$ is the velocity of this classical particle. Thus,

$$
\kappa_{p}(z)-\kappa_{P}(z)=\frac{\hbar k}{v_{p}(z)} .
$$

By substituting this formula in Eq. (5.12) and letting $P=p$ and $\kappa_{P}(z)=\kappa_{p}(z)$, because the differences $P-p$ and $\kappa_{P}(z)-\kappa_{p}(z)$ are of order $\hbar$, we find

$$
\begin{aligned}
\phi_{p^{\prime}}^{*}(z) \phi_{p}(z) & =\frac{p}{\kappa_{p}(z)} \exp \left[i k \int_{0}^{z} \frac{d z}{v_{p}(z)}\right] \\
& =\frac{p}{\kappa_{p}(z)} e^{i k t}
\end{aligned}
$$

where the time $t$ is defined by $d z / d t=v_{p}(z)$ with the condition $t=0$ at $z=0$. Hence

$$
\begin{aligned}
\mathcal{A}_{t}(\mathbf{p}, \mathbf{k}) & =-e \int_{-\infty}^{+\infty} \frac{d z}{v_{p}(z)} e^{i k t-i k^{z} z} \\
& =-e \int_{-\infty}^{+\infty} d t e^{i k t-i k^{z} z}
\end{aligned}
$$

It is convenient to define the variable $\xi$ by

$$
\begin{aligned}
\xi & \equiv t-z \cos \theta \\
\cos \theta & \equiv k^{z} / k
\end{aligned}
$$

Then

$$
\mathcal{A}_{t}(\mathbf{p}, \mathbf{k})=-e \int_{-\infty}^{+\infty} d \xi \frac{d t}{d \xi} e^{i k \xi}
$$

Next we turn our attention to the $z$-component given by Eq. (5.11), We note that, to lowest order in $\hbar$, we can let

$$
\frac{d \phi_{p}(z)}{d z}=i \frac{\kappa_{p}(z)}{\hbar} \phi_{p}(z)
$$

Hence

$$
\begin{aligned}
\mathcal{A}_{z}(\mathbf{p}, \mathbf{k}) & =e \int_{-\infty}^{+\infty} d z \frac{\kappa_{p}+\kappa_{P}}{2 p} \phi_{P}^{*}(z) \phi_{p}(z) e^{-i k^{z} z} \\
& =e \int_{-\infty}^{+\infty} d z e^{i k t-i k^{z} z} \\
& =e \int_{-\infty}^{+\infty} d \xi \frac{d z}{d \xi} e^{i k \xi}
\end{aligned}
$$

where we have let $\kappa_{P}(z)=\kappa_{p}(z)$ as before and used Eq. (5.15). This formula and Eq. (5.19) can be combined as

$$
\mathcal{A}^{\mu}(\mathbf{p}, \mathbf{k})=-e \int_{-\infty}^{+\infty} d \xi \frac{d x^{\mu}}{d \xi} e^{i k \xi}
$$

where $x^{\mu}$ is the classical trajectory with final momentum $p$ which passes through $(t, z)=(0,0)$. (The minus sign for the $z$-component is due to the raised index.) This 
emission amplitude is identical with that for a classical point charge passing through $(0,0)$ [11].

The expression (5.22) is ill-defined since $d x^{\mu} / d \xi$ remains finite as $\xi \rightarrow \pm \infty$. Therefore, we introduce a smooth cut-off function $\chi(\xi)$ which takes the value one while the acceleration is non-zero with the property $\lim _{\xi \rightarrow \pm \infty} \chi(\xi)=0$. Thus,

$$
\mathcal{A}^{\mu}(\mathbf{p}, \mathbf{k})=-e \int_{-\infty}^{+\infty} d \xi \frac{d x^{\mu}}{d \xi} \chi(\xi) e^{i k \xi} .
$$

In the end we take the limit $\chi(\xi) \rightarrow 1$ at any given $\xi$ in such a way that $\int_{-\infty}^{+\infty} d \xi\left[\chi^{\prime}(\xi)\right]^{2} \rightarrow 0$.

\section{DERIVATION OF THE LARMOR FORMULA}

In this section we derive the relativistic generalization of the Larmor formula for one-dimensional motion, Eq. (4.39), thus completing the demonstration of the equality $\delta z_{q 2}=\delta z_{\text {extra }}$. It is convenient to use the following form of the emission amplitude obtained by integration by parts:

$$
\mathcal{A}^{\mu}(\mathbf{p}, \mathbf{k})=-\frac{i e}{k} \int_{-\infty}^{+\infty} d \xi\left[\frac{d^{2} x^{\mu}}{d \xi^{2}}+\frac{d x^{\mu}}{d \xi} \chi^{\prime}(\xi)\right] e^{i k \xi},
$$

where we have used the condition that $\chi(\xi)=1$ if $d^{2} x^{\mu} / d \xi^{2} \neq 0$. By substituting this formula in Eq. (4.38) we obtain

$$
\begin{aligned}
\mathcal{E}_{\mathrm{em}}= & -e^{2} \int d \Omega \int_{0}^{\infty} \frac{d k}{16 \pi^{3}} \int_{-\infty}^{+\infty} d \xi^{\prime} \int_{-\infty}^{+\infty} d \xi \\
& \times\left[\frac{d^{2} x^{\mu}}{d \xi^{\prime 2}}+\frac{d x^{\mu}}{d \xi^{\prime}} \chi^{\prime}\left(\xi^{\prime}\right)\right] \\
& \times\left[\frac{d^{2} x_{\mu}}{d \xi^{2}}+\frac{d x_{\mu}}{d \xi} \chi^{\prime}(\xi)\right] e^{i k\left(\xi-\xi^{\prime}\right)}
\end{aligned}
$$

where $d \Omega$ is the solid angle in the $\mathbf{k}$-space. We extend the integration range for $k$ from $[0,+\infty)$ to $(-\infty,+\infty)$ and divide by two. Then using $\int_{-\infty}^{+\infty} e^{i k\left(\xi-\xi^{\prime}\right)} d k=2 \pi \delta\left(\xi-\xi^{\prime}\right)$, we find

$$
\begin{aligned}
\mathcal{E}_{\mathrm{em}}= & -\frac{e^{2}}{16 \pi^{2}} \int d \Omega \int_{-\infty}^{+\infty} d \xi \\
& \times\left\{\frac{d^{2} x_{\mu}}{d \xi^{2}} \frac{d^{2} x^{\mu}}{d \xi^{2}}+\frac{d x_{\mu}}{d \xi} \frac{d x^{\mu}}{d \xi}\left[\chi^{\prime}(\xi)\right]^{2}\right\} .
\end{aligned}
$$

The second term tends to zero in the limit $\chi(\xi) \rightarrow 1$ due to the requirement $\int_{-\infty}^{+\infty}\left[\chi^{\prime}(\xi)\right]^{2} d \xi \rightarrow 0$. Hence, we have in this limit

$$
\mathcal{E}_{\mathrm{em}}=-\frac{\alpha_{c}}{4 \pi} \int_{-\infty}^{+\infty} d \xi \int d \Omega \frac{d^{2} x_{\mu}}{d \xi^{2}} \frac{d^{2} x^{\mu}}{d \xi^{2}}
$$

where $\alpha_{c} \equiv e^{2} / 4 \pi$ as before. Now, one can readily show that

$$
\frac{d^{2} x^{\mu}}{d \xi^{2}}=\left(\frac{d t}{d \xi}\right)^{3}\left[\frac{d \xi}{d t} \frac{d^{2} x^{\mu}}{d t^{2}}-\frac{d^{2} \xi}{d t^{2}} \frac{d x^{\mu}}{d t}\right]
$$

By substituting $d \xi / d t=1-\dot{z} \cos \theta$ we find

$$
\begin{aligned}
\frac{d^{2} z}{d \xi^{2}} & =\frac{\ddot{z}}{(1-\dot{z} \cos \theta)^{3}}, \\
\frac{d^{2} t}{d \xi^{2}} & =\frac{d^{2} z}{d \xi^{2}} \cos \theta,
\end{aligned}
$$

and hence

$$
\frac{d^{2} x^{\mu}}{d \xi^{2}} \frac{d^{2} x_{\mu}}{d \xi^{2}}=-\frac{\ddot{z}^{2} \sin ^{2} \theta}{(1-\dot{z} \cos \theta)^{5}} \frac{d t}{d \xi} .
$$

By substituting this formula in Eq. (6.4) we obtain Eq. (4.39), thus demonstrating the equality $\delta z_{q 2}=$ $\delta z_{\text {extra}}$.

\section{QUANTUM POSITION SHIFT}

Our next task is to show that $\delta z_{q 1}=\delta z_{\mathrm{LD}}$. This will establish that the classical position shift $\delta z_{\text {class }}$ is equal to the quantum one $\delta z_{q}$ in the $\hbar \rightarrow 0$ limit.

In the product $\mathcal{A}^{\mu *}(\mathbf{p}, \mathbf{k}) \partial_{p} \mathcal{A}_{\mu}(\mathbf{p}, \mathbf{k})$ in Eq. 4.34) we use Eq. (6.1) for $\mathcal{A}^{\mu *}$ and Eq. (5.23) for $\partial_{p} \mathcal{A}_{\mu}$. Proceeding in a way similar to that led to Eq. (6.4) we find

$$
\begin{aligned}
\delta z_{q 1}=-\frac{\alpha_{c}}{4 \pi} & \int d \Omega \int d \xi\left\{\frac{d^{2} x^{\mu}}{d \xi^{2}} \frac{\partial}{\partial p}\left(\frac{d x^{\mu}}{d \xi}\right)\right. \\
& \left.+\frac{1}{4} \frac{\partial}{\partial p}\left(\frac{d x^{\mu}}{d \xi} \frac{d x_{\mu}}{d \xi}\right) \frac{d}{d \xi}[\chi(\xi)]^{2}\right\} .
\end{aligned}
$$

Next we demonstrate that the second term, which still contains the cut-off function, vanishes when integrated over the solid angle and $\xi$. Noting that

$$
\frac{d x^{\mu}}{d \xi} \frac{d x_{\mu}}{d \xi}=\left(\frac{d \tau}{d \xi}\right)^{2} \frac{d x^{\mu}}{d \tau} \frac{d x_{\mu}}{d \tau}=\left(\frac{d \tau}{d \xi}\right)^{2},
$$

we find by integration by parts that the contribution of this term is proportional to the following integral:

$$
I \equiv \int d \Omega \int_{-\infty}^{+\infty} d \xi \frac{d}{d \xi}\left\{\frac{\partial}{\partial p}\left(\frac{d \tau}{d \xi}\right)^{2}\right\}[\chi(\xi)]^{2} .
$$

Since $\partial / \partial p$ is taken with $\xi$ fixed, the $\xi$ - and $p$-derivatives commute. Hence this integral is equal to

$$
\begin{aligned}
I & =\frac{\partial}{\partial p} \int d \Omega \int_{-\infty}^{+\infty} d \xi \frac{d}{d \xi}\left(\frac{d \tau}{d \xi}\right)^{2}, \\
& =\frac{\partial}{\partial p}\left[\int d \Omega\left(\frac{d \tau}{d \xi}\right)^{2}\right]_{\xi=-\infty}^{\xi=+\infty},
\end{aligned}
$$

where we have used the fact that the $\xi$-derivative of $(d \tau / d \xi)^{2}$ is non-zero only if the acceleration is non-zero and, therefore, only when the cut-off function $\chi(\xi)$ equals one. Since

$$
\int d \Omega\left(\frac{d \tau}{d \xi}\right)^{2}=\frac{4 \pi}{(d t / d \tau)^{2}-(d z / d \tau)^{2}}=4 \pi,
$$


we have $I=0$, and thus the contribution from the second term in Eq. (7.1) vanishes. The remaining term gives the main contribution to the position shift due to radiation reaction in QED in the $\hbar \rightarrow 0$ limit as

$$
\delta z_{q 1}=-\frac{\alpha_{c}}{4 \pi} \int d \Omega \int d \xi \frac{d^{2} x^{\mu}}{d \xi^{2}} \frac{\partial}{\partial p}\left(\frac{d x_{\mu}}{d \xi}\right) .
$$

To compare $\delta z_{q 1}$ given by this equation with $\delta z_{\mathrm{LD}}$ given by Eq. (2.6) we need to find an expression of $\delta z_{q 1}$ in terms of $t$ rather than $\xi$. Thus, we need to eliminate the variable $\xi$ using its definition $\xi=t-z \cos \theta$. The two components of the second derivative $d^{2} x^{\mu} / d \xi^{2}$ are given by Eqs. (6.6) and (6.7). Let us consider the second factor of the integrand of (17.6), i.e. the $p$-derivative of $d x^{\mu} / d \xi$. By interchanging the order of integration we obtain

$$
\frac{\partial}{\partial p}\left(\frac{d x^{\mu}}{d \xi}\right)=\frac{d t}{d \xi} \frac{d}{d t}\left(\frac{\partial x^{\mu}}{\partial p}\right)_{\xi},
$$

where the subscript $\xi$ indicates that the partial derivative with respect to $p$ is taken with $\xi$ fixed. Then by differentiating the equation $t=z \cos \theta+\xi$ with respect to $p$ with $\xi$ fixed, we find

$$
\left(\frac{\partial t}{\partial p}\right)_{\xi}=\left(\frac{\partial z}{\partial p}\right)_{\xi} \cos \theta
$$

Combining this equation with Eqs. (6.6) and (6.7), which gives $d^{2} x^{\mu} / d \xi^{2}$, we can write the integrand in Eq. (7.6) as

$$
\frac{d^{2} x^{\mu}}{d \xi^{2}} \frac{\partial}{\partial p}\left(\frac{d x_{\mu}}{d \xi}\right)=-\frac{\ddot{z}}{(1-\dot{z} \cos \theta)^{4}} \frac{d}{d t}\left(\frac{\partial z}{\partial p}\right)_{\xi} \sin ^{2} \theta
$$

We substitute this expression in the quantum position shift (7.6) and change the integration variable from $\xi$ to $t$ and integrate by parts. The result is

$$
\begin{aligned}
\delta z_{q 1}=-\frac{\alpha_{c}}{4 \pi} \int & d \Omega \int_{-\infty}^{0} d t \frac{d}{d t}\left[\frac{\ddot{z}}{(1-\dot{z} \cos \theta)^{3}}\right] \\
& \times\left(\frac{\partial z}{\partial p}\right)_{\xi} \sin ^{2} \theta
\end{aligned}
$$

where we have also changed the integration range from $(-\infty,+\infty)$ to $(-\infty, 0]$ because $\ddot{z}=0$ for $[0,+\infty)$. Finally, we need to relate $(\partial z / \partial p)_{\xi}$ to $(\partial z / \partial p)_{t}$. By regarding $z$ as a function of $t$ and $p$ we have

$$
d z=\dot{z} d t+\left(\frac{\partial z}{\partial p}\right)_{t} d p
$$

By substituting $d t=d \xi+\cos \theta d z$ in this equation and rearranging, we obtain

$$
d z=\frac{\dot{z}}{1-\dot{z} \cos \theta} d \xi+\frac{1}{1-\dot{z} \cos \theta}\left(\frac{\partial z}{\partial p}\right)_{t} d p .
$$

Thus,

$$
\left(\frac{\partial z}{\partial p}\right)_{\xi}=\frac{1}{1-\dot{z} \cos \theta}\left(\frac{\partial z}{\partial p}\right)_{t}
$$

Using this expression in the position shift (7.10) we have

$$
\begin{aligned}
\delta z_{q 1}=-\frac{\alpha_{c}}{4 \pi} & \int d \Omega \int_{-\infty}^{0} d t\left(\frac{\partial z}{\partial p}\right)_{t} \\
& \times\left[\frac{d \ddot{z}}{d t} \frac{\sin ^{2} \theta}{(1-\dot{z} \cos \theta)^{4}}+3 \ddot{z}^{2} \frac{\sin ^{2} \theta \cos \theta}{(1-\dot{z} \cos \theta)^{5}}\right] .
\end{aligned}
$$

We can now perform the integration over the solid angle with the following result:

$$
\begin{aligned}
\delta z_{q 1} & =-\frac{2 \alpha_{c}}{3} \int_{-\infty}^{0} d t\left[\gamma^{4} \frac{d \ddot{z}}{d t}+3 \gamma^{6} \ddot{z}^{2} \dot{z}\right]\left(\frac{\partial z}{\partial p}\right)_{t} \\
& =-\int_{-\infty}^{0} d t\left[\frac{2 \alpha_{c}}{3} \gamma \frac{d}{d t}\left(\gamma^{3} \ddot{z}\right)\right]\left(\frac{\partial z}{\partial p}\right)_{t} \\
& =-\int_{-\infty}^{0} d t F_{\mathrm{LD}}\left(\frac{\partial z}{\partial p}\right)_{t},
\end{aligned}
$$

where we have used the expression of $F_{\mathrm{LD}}$ given by Eq. (2.3). This formula will be used in the next section to show that $\delta z_{q 1}=\delta z_{\mathrm{LD}}$. We note here that Eq. (7.15) is valid for an external force of any form provided that the photon emission amplitude is given by Eq. (5.22).

\section{COMPARISON OF CLASSICAL AND QUANTUM POSITION SHIFTS}

Comparison between Eqs. (7.15) and (2.6) shows that the equality $\delta z_{q 1}=\delta z_{\mathrm{LD}}$ follows if

$$
\left(\frac{\partial z}{\partial p}\right)_{t}=\frac{v_{0}}{m} \dot{z}(t) \int_{0}^{t} \frac{d t^{\prime}}{\gamma^{3}\left(t^{\prime}\right)\left[\dot{z}\left(t^{\prime}\right)\right]^{2}} d t^{\prime} .
$$

This equation can be demonstrated as follows. Since the energy is conserved, we have

$$
\sqrt{m^{2}(d z / d \tau)^{2}+m^{2}}+V(z)=\sqrt{p^{2}+m^{2}},
$$

and, hence,

$$
\frac{d z}{d t}=\left[1-m^{2}\left(\sqrt{p^{2}+m^{2}}-V(z)\right)^{-2}\right]^{1 / 2} .
$$

By differentiating both sides with respect to $p$ with $t$ fixed, and noting that

$$
\begin{aligned}
p / \sqrt{p^{2}+m^{2}} & =v_{0}, \\
\sqrt{p^{2}+m^{2}}-V(z) & =m \gamma,
\end{aligned}
$$

we obtain

$$
\frac{d}{d t}\left(\frac{\partial z}{\partial p}\right)_{t}=\frac{1}{m \gamma^{3} \dot{z}}\left[v_{0}-V^{\prime}(z)\left(\frac{\partial z}{\partial p}\right)_{t}\right] .
$$

By substituting the formula $V^{\prime}(z)=-m \gamma^{3} \ddot{z}$ [see Eq. (2.5)] in Eq. (8.6) we find

$$
\frac{d}{d t}\left[\frac{1}{\dot{z}}\left(\frac{\partial z}{\partial p}\right)_{t}\right]=\frac{v_{0}}{m \gamma^{3} \dot{z}^{2}}
$$


Then by integrating this formula, remembering that $z=$ 0 at $t=0$ for all $p$, we arrive at Eq. (8.1).

The derivation of $\delta z_{q 1}=\delta z_{\mathrm{LD}}$ above uses an explicit relation, Eq. (8.3), between the the trajectory $z(t)$ and the momentum $p$. However, the simple form for the quantum position shift $\delta z_{q 1}$ given by Eq. (7.15) suggests that there should be a derivation which does not rely on the relation (8.3). We show next that there is indeed such a derivation which is valid in a more general setting.

We consider a charged particle moving in the $z$ direction accelerated by an external force which may depend on both $z$ and $t$. Thus, we consider the following equation of motion:

$$
m \frac{d^{2} z}{d \tau^{2}}=\left[F_{\mathrm{ext}}(t, z)+F_{\mathrm{LD}}\right] \frac{d t}{d \tau} .
$$

This equation describes a classical particle under the influence of an external force $F_{\text {ext }}(t, z)$ and the LorentzDirac force. The model analyzed so far in this paper is the special case given by $F_{\text {ext }}(t, z)=-V^{\prime}(z)$.

We first consider a solution to Eq. (8.8) in the absence of radiation reaction, i.e. with $F_{\mathrm{LD}}$ set to 0 , with the momentum and the position being $p$ and 0 , respectively, at $t=0$. We define $P \equiv m d z / d \tau$ and let this solution be denoted

$$
(z, P)=(\bar{z}(t), \bar{P}(t)) .
$$

We have $(\bar{z}(0), \bar{P}(0))=(0, p)$. Let a linearized solution about $(\bar{z}, \bar{P})$, also with $F_{\mathrm{LD}}=0$, be given by

$$
(z, P)=(\bar{z}+\Delta z, \bar{P}+\Delta P) .
$$

From the equation of motion (with $F_{\mathrm{LD}}=0$ ) the quantities $\Delta z$ and $\Delta P$ are found to satisfy the equations

$$
\begin{aligned}
\frac{d}{d t} \Delta z & =m^{-1}\left(1-\dot{\bar{z}}^{2}\right)^{3 / 2} \Delta P \equiv A(t) \Delta P \\
\frac{d}{d t} \Delta P & =\left.\frac{\partial F_{\text {ext }}}{\partial z}\right|_{z=\bar{z}} \Delta z \equiv B(t) \Delta z
\end{aligned}
$$

Let $\left(\Delta z_{s}(t), \Delta P_{s}(t)\right)$ be a set of solutions labeled by $s$ of these equations satisfying $\left(\Delta z_{s}(s), \Delta P_{s}(s)\right)=(0,1)$. The linearized solutions represent a particle whose position at $t=0$ coincides with $\bar{z}(0)$ (see Fig. 3 ). The quantity $(\partial z / \partial p)_{t}$ in Eq. (7.15) is the rate of change in the position $z$ at time $t$ as the momentum $P$ at $t=0$ is changed while the position $z$ at $t=0$ is fixed. Thus,

$$
\left(\frac{\partial z}{\partial p}\right)_{t}=\frac{\Delta z_{0}(t)}{\Delta P_{0}(0)}=\Delta z_{0}(t) .
$$

By substituting this formula in Eq. (7.15) we obtain

$$
\delta z_{q 1}=-\int_{-\infty}^{0} d t F_{\mathrm{LD}}(t) \Delta z_{0}(t) .
$$

Next a similar expression will be derived for $\delta z_{\mathrm{LD}}$. We again let $(\bar{z}(t), \bar{P}(t))$ be a solution of (8.8) with $F_{\mathrm{LD}}=0$,

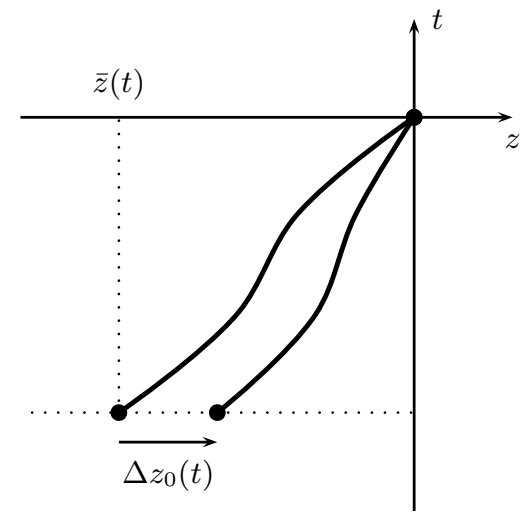

FIG. 3: The world lines for the solutions $(\bar{z}(t), \bar{P}(t))$ and $\left(\bar{z}(t)+\Delta z_{0}(t), \bar{P}(t)+\Delta P_{0}(t)\right)$

and now let $(\bar{z}+\delta z, \bar{P}+\delta P)$ be the approximate solution obtained by perturbing $(\bar{z}, \bar{P})$ to first order in $F_{\mathrm{LD}}$ with the initial conditions $(\delta z, \delta P)=(0,0)$ before the acceleration starts. The quantities $\delta z$ and $\delta P$ satisfy

$$
\begin{aligned}
\frac{d}{d t} \delta z & =A(t) \delta P, \\
\frac{d}{d t} \delta P & =B(t) \delta z+F_{\mathrm{LD}}(t),
\end{aligned}
$$

where $A(t)$ and $B(t)$ are defined by Eqs. (8.11) and (8.12), respectively. The quantity $\delta z(\delta P)$ is the difference in position (momentum) between the hypothetical non-radiating particle and the radiating particle. Thus, the quantity $\delta z$ at $t=0$ is equal to $\delta z_{\mathrm{LD}}$.

The solutions to Eqs. 8.15) and (8.16) with the initial conditions specified above can be expressed in terms of another set of solutions $\left(\Delta z_{s}(t), \Delta P_{s}(t)\right)$ of the nonradiating equations, Eqs. (8.11) and (8.12), as

$$
\begin{aligned}
\delta z(t) & =\int_{-\infty}^{t} d s F_{\mathrm{LD}}(s) \Delta z_{s}(t), \\
\delta P(t) & =\int_{-\infty}^{t} d s F_{\mathrm{LD}}(s) \Delta P_{s}(t) .
\end{aligned}
$$

These can readily be shown to satisfy Eqs. 8.15 and (8.16) by direct differentiation using the conditions $\Delta_{t} z(t)=0$ and $\Delta_{t} P(t)=1$. The classical position shift due to the Lorentz-Dirac force, at time $t=0$, is therefore given by

$$
\delta z_{\mathrm{LD}}=\int_{-\infty}^{0} d t F_{\mathrm{LD}}(t) \Delta z_{t}(0) .
$$

This expression for the classical position shift $\delta z_{\mathrm{LD}}$ is similar to that for the quantum position shift $\delta z_{q 1}$ in Eq. (8.14), the only difference being that the quantity $\Delta z_{0}(t)$ is replaced by $-\Delta z_{t}(0)$. In Fig. 4 we show the world lines corresponding to the solution $(\bar{z}(s), \bar{P}(s))$ and 


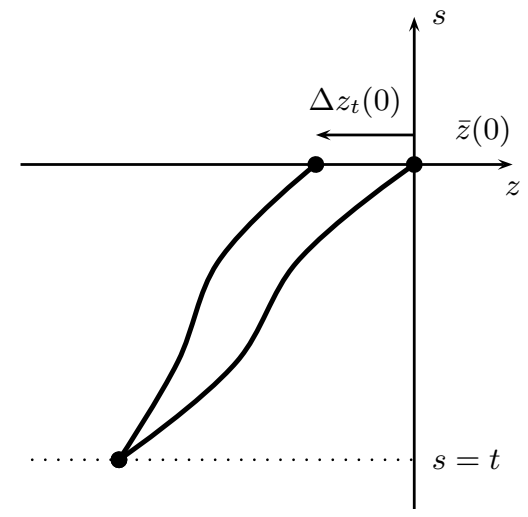

FIG. 4: The world lines for the solutions $(\bar{z}(s), \bar{P}(s))$ and $\left(\bar{z}(s)+\Delta z_{t}(s), \bar{P}(s)+\Delta P_{t}(s)\right)$

the approximate solution $\left(\bar{z}(s)+\Delta z_{t}(s), \bar{P}(s)+\Delta P_{t}(s)\right)$ to the equations for the hypothetical non-radiation particle motion.

Now, we only need to establish that $\Delta z_{0}(t)=-\Delta z_{t}(0)$ to show that the quantum and classical position shifts (8.14) and (8.19) are equal. In fact one can show in general that $\Delta z_{s}(t)=-\Delta z_{t}(s)$ for any values of $s$ as follows. Given a pair of solutions $\left(\Delta z^{(1)}, \Delta P^{(1)}\right)$ and $\left(\Delta z^{(2)}, \Delta P^{(2)}\right)$ to Eqs. (8.11) and (8.12) we define the symplectic product by

$$
\begin{aligned}
& \left\langle\Delta z^{(1)}, \Delta P^{(1)} \mid \Delta z^{(2)}, \Delta P^{(2)}\right\rangle \\
& \equiv \Delta z^{(1)} \Delta P^{(2)}-\Delta P^{(1)} \Delta z^{(2)} .
\end{aligned}
$$

It can readily be shown that the symplectic product is time-independent by differentiating it with respect to $t$ and using Eqs. (8.11) and (8.12). With the identification $\left(\Delta z^{(1)}, \Delta P^{(1)}\right)=\left(\Delta z_{s}, \Delta P_{s}\right)$ and $\left(\Delta z^{(2)}, \Delta P^{(2)}\right)=$ $\left(\Delta z_{t}, \Delta P_{t}\right)$ we obtain

$$
\begin{aligned}
& \Delta z_{s}(t) \Delta P_{t}(t)-\Delta P_{s}(t) \Delta z_{t}(t) \\
& =\Delta z_{s}(s) \Delta P_{t}(s)-\Delta P_{s}(s) \Delta z_{t}(s) .
\end{aligned}
$$

Since $\Delta z_{s}(s)=\Delta z_{t}(t)=0$ and $\Delta P_{s}(s)=\Delta P_{t}(t)=1$, we have $\Delta z_{s}(t)=-\Delta z_{t}(s)$. Hence the equality $\delta z_{q 1}=$ $\delta z_{\mathrm{LD}}$ holds. This equality and the equality $\delta z_{q 2}=\delta z_{\text {extra }}$ establish $\delta z_{q}=\delta z_{\text {class }}$ [see Eqs. (2.7) and (4.33)]. Thus, the position shift due to QED to order $e^{2}$ in the limit $\hbar \rightarrow 0$ is equal to the position shift due to the LorentzDirac force for linear motion if the acceleration is caused by a static potential.

We have shown that the classical position shift $\delta z_{\mathrm{LD}}$ equals $\delta z_{q 1}$ in Eq. 8.14 for any external force which depends on $t$ and $z$. We have also shown that Eq. 8.14 is valid provided that the photon emission amplitude is given by $E q$. (5.22). Thus, the equality $\delta z_{q 1}=\delta z_{\mathrm{LD}}$ holds if the amplitude $\mathcal{A}^{\mu}(\mathbf{p}, \mathbf{k})$ is given by Eq. (5.22). We have demonstrated Eq. (5.22) for the case where the external force $F_{\text {ext }}$ is $t$-independent. The extension to the case where the external force depends on both $t$ and $z$ does not appear to be straightforward. However, our method can be applied to the case with an external force that depends only on $t$ because the WKB approximation is very similar to that for the $t$-independent case as we demonstrate in the next section.

\section{TIME-DEPENDENT POTENTIAL}

Let the potential be given by $V_{t}=0, V_{z}=-V(t)$ in the QED Lagrangian density (3.1). We show below that the emission amplitude in this case is also given by Eq. (5.22).

Since the calculation is quite similar to the timeindependent case, we present only a brief description. The WKB approximation to the mode functions is given by

$$
\begin{aligned}
\Phi_{\mathbf{p}}(t, \mathbf{x}) & \approx \tilde{\phi}_{p}(t) \exp \left[\frac{i}{\hbar}\left(\mathbf{p}_{\perp} \cdot \mathbf{x}_{\perp}+p^{z} z\right)\right], \\
\tilde{\phi}_{p}(t) & \equiv \sqrt{\frac{p_{0}}{\sigma_{p}(t)}} \exp \left[-\frac{i}{\hbar} \int_{0}^{t} \sigma_{p}(\zeta) d \zeta\right]
\end{aligned}
$$

where

$$
\sigma_{p}(t) \equiv \sqrt{[p-V(t)]^{2}+m^{2}}
$$

if $\mathbf{p}_{\perp}=0$. (The mass term $m^{2}$ needs to be replaced by $m^{2}+\mathbf{p}_{\perp}^{2}$ if $\mathbf{p}_{\perp} \neq 0$ as before.) The function $\sigma_{p}(t)$ is the $t$-component of the kinetic energy-momentum $m d x^{\mu} / d \tau$ of the non-radiating classical particle at $t$. Note that the momentum $\mathbf{p}$ including the $z$-component $p^{z}=p$ is conserved, but the energy is not. The calculation of the emission amplitude follows the same pattern as before with the roles of $t$ and $z$ (and those of $p$ and $p_{0}$ ) reversed.

Let $\mathcal{A}_{\mu}(\mathbf{p}, \mathbf{k})$ be, again, the emission amplitude for the process in which the charged particle with momentum $\mathbf{p}$ emits a photon with momentum $\hbar \mathbf{k}$ so that the final momentum of the charged particle is given by $\mathbf{P}=\mathbf{p}-\hbar \mathbf{k}$. We again assume that the transverse momenta are of order $\hbar$ and can be neglected. Then the $z$-component to order $\hbar^{0}$ [to be compared with Eq. (5.10)] is

$$
\mathcal{A}_{z}=e \int d t \frac{p-V(t)}{p_{0}} \tilde{\phi}_{P}^{*}(t) \tilde{\phi}_{p}(t) e^{i k t} .
$$

For the $t$-component [to be compared with Eq. [5.11]] we obtain

$$
\mathcal{A}_{t}=-\frac{i e \hbar}{2 p_{0}} \int d t\left[\tilde{\phi}_{P}^{*} \partial_{t} \tilde{\phi}_{p}-\left(\partial_{t} \tilde{\phi}_{P}^{*}\right) \tilde{\phi}_{p}\right] e^{i k t} .
$$

The product of mode functions in Eq. (9.4) is

$$
\begin{aligned}
\tilde{\phi}_{P}^{*}(t) \tilde{\phi}_{p}(t)= & \sqrt{\frac{P_{0} p_{0}}{\sigma_{P}(t) \sigma_{p}(t)}} \\
& \times \exp \left[-\frac{i}{\hbar} \int_{0}^{t}\left(\sigma_{p}(\zeta)-\sigma_{P}(\zeta)\right) d \zeta\right] .
\end{aligned}
$$


To lowest order in $\hbar$ we can change $P_{0}$ to $p_{0}$ and $\sigma_{P}(t)$ to $\sigma_{p}(t)$ in the pre-factor as the differences are of order $\hbar$. Recalling the classical momentum conservation equation, $m d z / d \tau+V(t)=p$, and the formula $m d t / d \tau=\sigma_{p}(t)$, where $z(t)$ is the classical trajectory with $z(0)=0$, we obtain to lowest order in $\hbar$

$$
\sigma_{p}(t)-\sigma_{P}(t)=\frac{\partial \sigma_{p}}{\partial p}(p-P)=\frac{d z}{d t} \hbar k^{z} .
$$

Thus the product of the mode functions in question can be written

$$
\begin{aligned}
\tilde{\phi}_{P}^{*}(t) \tilde{\phi}_{p}(t) & =\frac{p_{0}}{\sigma_{p}} \exp \left[-\frac{i}{\hbar} \int_{0}^{t} d t \frac{d z}{d t} \hbar k^{z}\right] \\
& =\frac{p_{0}}{\sigma_{p}} \exp \left(-i k^{z} z\right) .
\end{aligned}
$$

This gives the $z$-component of the emission amplitude as

$$
\begin{aligned}
\mathcal{A}_{z}(\mathbf{p}, \mathbf{k}) & =e \int d t \frac{p-V(t)}{\sigma_{p}(t)} e^{i k t-i k^{z} z} \\
& =e \int d \xi \frac{d z}{d \xi} e^{i k \xi},
\end{aligned}
$$

where $\xi=t-z \cos \theta$ and $\cos \theta \equiv k^{z} / k$ as before. To calculate the $t$-component of the amplitude, note first that to lowest order in $\hbar$ we have

$$
\begin{aligned}
\hbar \tilde{\phi}_{p^{\prime}}(t) \partial_{t} \tilde{\phi}_{p}(t) & =-i \sigma_{p}(t) \tilde{\phi}^{*}(t) \tilde{\phi}_{p}(t) \\
& =-i p_{0} \exp \left(-i k^{z} z\right)
\end{aligned}
$$

where Eq. (9.8) has been used. By using this equation in Eq. 9.5 we obtain

$$
\mathcal{A}_{t}(\mathbf{p}, \mathbf{k})=-e \int d t e^{i k t-i k^{z} z}=-e \int d \xi \frac{d t}{d \xi} e^{i k \xi} .
$$

Eqs. (9.9) and 9.11 can be written $\mathcal{A}^{\mu}(\mathbf{p}, \mathbf{k})=$ $-e \int d \xi\left(d x^{\mu} / d \xi\right) e^{i k \xi}$. As we mentioned before this equality is sufficient to conclude that $\delta z_{q 1}=\delta z_{\mathrm{LD}}$.

The equality $\delta z_{q 2}=\delta z_{\text {extra }}$, which is necessary to establish the equality of the classical and quantum position shift, is trivial in this case. Since the system is translationally invariant, the classical position shift is invariant under the shift in the position, $\langle z\rangle=0 \rightarrow z_{0}$, of the non-radiation hypothetical particle at $t=0$. Hence $\delta z_{\text {extra }}=0$. Furthermore, since $P=p-\hbar k^{z}$, we have $d p / d P=1$ and hence $\delta z_{q 2}=0$ in Eq. 4.35). Thus, we have $\delta z_{q 2}=\delta z_{\text {extra }}=0$. Hence, $\delta z_{q}=\delta z_{\text {class }}$, i.e. the position shift from the Lorentz-Dirac force agrees with that in QED in the limit $\hbar \rightarrow 0$ to order $e^{2}$ for a spaceindependent potential as well.

The equality $\delta z_{q 1}=\delta z_{\mathrm{LD}}$ can be verified by a more explicit calculation as in the case with a static potential. Note that the momentum conservation equation reads

$$
\frac{d}{d t}[m \gamma \dot{z}+V(t)]=F_{\mathrm{LD}} .
$$

Hence

$$
\delta(m \gamma \dot{z})=m \gamma^{3} \frac{d}{d t}(\delta z)=\int_{-\infty}^{t} F_{\mathrm{LD}}\left(t^{\prime}\right) d t^{\prime} .
$$

Thus, the classical position shift is

$$
\delta z_{\text {class }}=\delta z_{\mathrm{LD}}=-\int_{-\infty}^{0}\left(\int_{0}^{t} \frac{1}{m \gamma^{3}} d t^{\prime}\right) F_{\mathrm{LD}} d t
$$

where we have interchanged the order of integration. Thus, what we need to establish is

$$
\left(\frac{\partial z}{\partial p}\right)_{t}=\int_{0}^{t} \frac{1}{m \gamma^{3}} d t
$$

for the hypothetical non-radiating particle. The momentum conservation for this particle in the $z$-direction reads

$$
m \frac{d z}{d \tau}+V(t)=p .
$$

Hence, with the condition $z=0$ at $t=0$, we find

$$
z=\int_{0}^{t}\left\{1+\frac{m^{2}}{[p-V(t)]^{2}}\right\}^{-1 / 2} d t
$$

By differentiating this expression with respect to $p$ and using $p-V(t)=m d z / d \tau$ and $\sqrt{[p-V(t)]^{2}+m^{2}}=$ $m d t / d \tau$, we indeed obtain Eq. (9.15).

\section{CONCLUSION}

In this paper we showed that the change in position due to the radiation reaction of a particle, which we call the position shift, according to the Lorentz-Dirac theory in classical electrodynamics is reproduced by the $\hbar \rightarrow 0$ limit of QED with a scalar charged particle. The calculation was performed to lowest non-trivial order in $e^{2}$ for wave packets linearly accelerated for a finite time by either a purely space-dependent or time-dependent potential. The quantum wave functions constituting the wave packets were approximated using the WKB functions for each potential. The agreement between QED and the Lorentz-Dirac theory was demonstrated by concrete calculations which gave the position shifts explicitly. We also showed by a general argument that this agreement holds as long as the emission amplitude coincides in the $\hbar \rightarrow 0$ limit with that for a classical point charge.

This work extended the corresponding work in the nonrelativistic approximation 9, 10 and provided the details omitted in Ref. [11] as well as the analysis for the timedependent but space-independent potential. It will be interesting to generalize our results to the case with a particle moving in three space dimensions. It is also important to estimate the one-loop correction to the potential. In the (unrealistic) model in which the acceleration is achieved by a space-dependent mass term [10], this 
correction is of order $\hbar^{-1}$, thus overwhelming the contribution from the Lorentz-Dirac force. If this correction turns out to be of order $\hbar^{0}$ in the models studied here, which are more realistic, then the one-loop correction will be as important as the Lorentz-Dirac force in determining the motion of the charged particle. It will also be interesting to see whether our results can be justified when more than one photons are emitted: our results, as they stand, are logically consistent only if the emission probability $\mathcal{P}_{\text {em }}$ given by Eq. (4.23) is much smaller than one so that the process is dominated by one-photon emission. These issues are currently under investigation.
[1] M. Abraham and R. Becker, Theorie der Elektrizität (Springer, Leipzig, 1933), Vol. II.

[2] H. A. Lorentz, Theory of electrons, (Dover, New York, 1952).

[3] P. A. M. Dirac, Proc. Roy. Soc. London A167, 148 (1938).

[4] E. Poisson, An introduction to the Lorentz-Dirac equation, ArXiv:gr-qc/9912045

[5] C. Teitelboim, Phys. Rev. D 1, 1572 (1970); 3, 297 (1971); 4, 345 (1971).

[6] J. D. Jackson, Classical electrodynamics, (Wiley, New York, 1975)

[7] F. Rohrlich, Classical charged particles, (Addison-Wesley Publishing, Massachusetts, 1965)

[8] L. D. Landau and E. M. Lifshitz, The classical theory of fields, (Pergammon, Oxford, 1962).
[9] A. Higuchi, Radiation reaction in quantum mechanics, arXiv:quant-ph/9812036

[10] A. Higuchi, Phys. Rev. D 66, 105004 (2002); 69, 129903(E) (2004), arXiv:quant-ph/0208017

[11] A. Higuchi and G. D. R. Martin, arXiv:quant-ph/0407162 Phys. Rev. D 70, 081701(R) (2004).

[12] E. J. Moniz and D. H. Sharp, Phys. Rev. D 10, 1133, (1974); 15, 2850, (1977).

[13] P. R. Johnson and B. L. Hu, Phys. Rev. D 65, 065015 (2002), ArXiv: quant-ph/0101001

[14] V. S. Krivitskiî and V. N. Tsytovich, Sov. Phys. Usp. 34, 250 (1991).

[15] R. Shankar, Principles of quantum mechanics, (Plenum, New York, 1994), pp. 435-438. 